\title{
Habilidades del pensamiento y rendimiento académico en estudiantes universitarios de las carreras de Ingeniería y Arquitectura
}

\section{Skills of the Thought and Academic Performance in university students of the careers of Engineering and Architecture}

\author{
Rosario Arias Barahona Guzmán ${ }^{1}$ \\ Instituto Peruano de Psicología y Educación
}

\author{
Ana Sofía Aparicio Pereda ${ }^{2}$ \\ Universidad de Sao Paulo
}

Recibido: $30-05-18$

Aceptado: $23-07-18$

\section{Resumen}

El estudio buscó establecer la relación entre las habilidades del pensamiento y de sus factores con el rendimiento académico en un grupo de ingresantes universitarios de las carreras de Ingeniería y Arquitectura. La muestra total fue de 113 participantes, con la que se hicieron todos los análisis referentes a las habilidades del pensamiento, distribución de factores y niveles. El análisis de relación entre rendimiento académico y habilidades del pensamiento se realizó con 98 participantes que contaban con los promedios finales de notas. Los resultados muestran que los participantes se distribuyen mayoritariamente en el nivel Normal Inferior al Normal y la existencia de una asociación positiva moderada con el rendimiento académico. No se encontraron diferencias de acuerdo al género, edad y/o especialidad.

Palabras clave: Habilidades del pensamiento; rendimiento académico; ingresante universitario.

\begin{abstract}
The study sought to establish the relationship between the skills of thinking and their factors with academic performance in a group of university entrantsof the careers of engineering and architecture. The total sample was of 113 participants, with whom all the analyzes referring tothinking skills, distribution of factors and levels were carried out.The analysis of the relationship between performance and thinking skills was carried out with 98 participants who counted on the final averages of the ratings. The results show that the participants are distributed mostly in the Normal Lower an3d Normal level and the existence of a moderate positive association with the academic performance. No differences were found according to gender, age and /or specialty.
\end{abstract}

Keywords: Thinking skills; academic performance; university entrant.

\footnotetext{
1 Presidenta del Instituto Peruano de Psicología y Educación. Autora para correspondencia.

E-mail: rosario.arias@psicologiayeducacion.org

2 Docente Universidad de Sao Paulo. Email: anasofiap@usp.br
}

(C) Los autores. Este artículo es publicado por la Revista de Investigación en Psicología de la Facultad de Psicología, Universidad Nacional Mayor de San Marcos. Este es un artículo de acceso abierto, distribuido bajo los términos de la licencia Creative Commons Atribucion - No Comercia_Compartir Igual 4.0 Internacional. (http://creativecommons.org/licenses/by-nc-sa/4.0/) que permite el uso no comercial, distribución y reproducción en cualquier medio, siempre que la obra original sea debidamente citada. 


\section{INTRODUCCIÓN}

La presente investigación se aborda el asunto de las habilidades del pensamiento, aquellas que posibilitan construir y organizar el conocimiento, para utilizarlo con eficacia en diversas circunstancias en las que hay que resolver problemas, habilidades que se desarrollan con la práctica y se relacionan con el acto de pensar (Velásquez, Remolina y Calle, 2014).

La fundamentación teórica de los modelos para evaluar y desarrollar las habilidades del pensamiento son las teorías acerca del funcionamiento cognitivo y la estimulación de sus procesos. Los autores que representan el enfoque cognitivo son: Gardner (1983); Goleman (1986), Sánchez (1985, 1992, 2002) y Sternberg (1985, 1987), y con su paradigma de procesos así como a Feuerstein (1997) con su modelo de modificabilidad cognitiva estructural (Citado por Tapia y Luna, 2010).

La teoría de modificabilidad cognitiva estructural (Feuerstein, 1990, 1997) aborda la inteligencia, entendida como pasible de ser modificada estructuralmente, como respuesta a las necesidades de adaptación a estímulos internos o externos nuevos; dicha adaptabilidad es esencial para la resolución de problemas y comprende componentes cognitivos, motivacionales y creativos. Tal adaptabilidad demanda de nuevas estructuras cognitivas que constituyen elementos afectivos de la personalidad.

Según Sánchez (2002), la mente es entendida como un sistema representacional del mundo y que hace posible estructurar el conocimiento. Entre estos procesos tenemos el pensamiento.

Feuerstein $(1990,1997)$, señala que las operaciones cognitivas (acciones interiorizadas) suponen la manipulación y combinaciones de representaciones, icónicas, conceptuales, lingüísticas.

Las operaciones y funciones del pensamiento que se han identificado, son:

\section{Cuadro 1}

Operaciones mentales de acuerdo a la teoría de Feurstein

\begin{tabular}{llll}
\hline Razonamiento lógico & Razonamiento hipotético & $\begin{array}{l}\text { Proyección de Relaciones } \\
\text { virtuales }\end{array}$ & Transformación mental \\
\hline Pensamiento divergente & Razonamiento analógico & Codificación-decodificación & Representación mental \\
\hline Razonamiento silogístico & Inferencia lógica & Clasificación & Diferenciación \\
\hline Razonamiento transitivo & Análisis-síntesis & Comparación & Identificación \\
\hline
\end{tabular}

Fuente: Feuerstein (1997) en Tapia y Luna (2010)

Siguiendo a Feuerstein $(1990,1997)$, las funciones cognitivas se agrupan en tres categorías, funciones (a) de entrada, utilizadas en la búsqueda de información, (b) de elaboración, utilizadas en el procesamiento de la información y, (c) cognitivas de salida, usadas para expresar la respuesta o resultado del acto mental. 
Los procesos del pensamiento, Sánchez (2002) los clasifica en: (a) procesos básicos (de construcción de conocimientos, de organización del conocimiento, de integración y juicio crítico); (b) procesos superiores (directivos, ejecutivos para el manejo de la información, de adquisición de conocimientos y discernimiento); (c) procesos de razonamiento (deductivo, inductivo, hipotético y analógico) y (d) procesos creativos (expansión y contracción de ideas, extensión de campo y activación cognitiva e inventiva).

En la actualidad, se propugna por una educación que se centre en el enfoque cognitivista (Muelas y Navarro, 2014), en la que el protagonista de del aprendizaje sea el estudiante; para lo cual es importante los educadores consideren la importancia del cerebro humano y el conocimiento de su funcionamiento, y podrían ayudar a sus estudiantes a través de estrategias de aprendizaje, que los harían pensadores efectivos, reflexivos, creativos y críticos, capaces de planificar y reflexionar sobre su actividad.

Las habilidades del pensamiento son la base sobre los que se construye y organiza el conocimiento y el razonamiento. Su desarrollo propicia un aprendizaje más duradero, significativo y aplicable en la toma de decisiones y en la solución de problemas cotidianos.

De acuerdo con Velásquez, Remolina y Calle $(2013$, p. 39) si se pretende formar personas críticas, autónomas, reflexivas y creativas, es importante contribuir al desarrollo de las habilidades de pensamiento, que les permita resolver problemas, explicar situaciones desde diferentes áreas del saber, manejar información, participar en actividades colaborativas, innovar y crear en diversas circunstancias de la vida.

A partir de la idea según la cual la educación universitaria debe plantearse como objetivos, el desarrollo de habilidades del pensamiento y la estimulación del pensamiento creativo, consideramos imperativo que las autoridades universitarias y profesores de todas los especialidades consideren de la mayor importancia generar propuestas educativas que promuevan el desarrollo de habilidades de pensamiento crítico y creativo en los educandos que conlleven a potenciar el aprendizaje.

El pensamiento crítico y la creatividad son fortalezas psicológicas que todas los seres humanos poseemos, están en estado virtual listos para ser potenciados, los que junto a la curiosidad, ganas de aprender y perspectiva, se constituyen en fuerzas cognitivas para la adquisición y aplicación del conocimiento.

La presente investigación de tipo descriptivo-comparativa y correlacional,se propusorelacionar los niveles de desarrollo global de las habilidades y sus factores con el rendimiento académico evaluado al final del semestre académico; así como identificar los niveles de desarrollo de las habilidades de pensamiento a nivel global y en sus factores, que caracterizan aun grupo de estudiantes de ingeniería 
y arquitectura de la Facultad de Ingeniería y Arquitectura de una universidad privada de Lima. Asimismo, se buscó establecer las diferencias existentes según género, edad y especialidad.

\section{MÉTODO}

\section{Participantes}

En el estudio participaron 125 ingresantes universitarios de las carreras de ingeniería y arquitectura, de los cuales se obtuvo una muestra final de 113 participantes, 62 varones $(54.9 \%$ ) y 51 mujeres (45.1\%), que pasaron las mediciones pre y post. De los 113 participantes solo 98 participantes contaron con notas finales, con este último grupo se realizó el análisis de relación entre habilidades del pensamiento y rendimiento académico.

Al término del semestre 2017-I, para efectos de las correlaciones que se efectuaron entre las habilidades del pensamiento y sus factores con el rendimiento académico, la muestra se redujo a 98 estudiantes que fueron los que cumplimentaron las evaluaciones en todas las asignaturas; un $22 \%$ cursó menos asignaturas de las programadas para el primer ciclo o se retiró de la facultad.

La selección de la muestra fue de tipo intencional, obtenida en la Facultad de Ingeniería y Arquitectura de una Universidad privada de Lima, en el marco de un curso introductorio de nivelación ofrecido a los jóvenes ingresantes semestre 2017- I, previo al inicio del primer ciclo.

\section{Instrumento}

\section{Prueba de Habilidades del Pensamiento (PHP)}

La Prueba de Habilidades del Pensamiento fue elaborada por Tapia y Luna (2010), para ser utilizada en estudiantes de secundaria de cuarto, quinto año y de primer año de universidad. Consta de un total de 41 ítems, distribuidos en los diferentes factores que componen la prueba y se refieren a enunciados, que conciernen a planteamientos de un problema o a textos, seguido cada uno de ellos de una o más preguntas con cuatro alternativas de respuesta. Se aplica en 75 minutos como máximo, de forma individual o grupal y cuenta con normas percentiles.

Los factores de la prueba son 1. Inferencia lógica deductiva e inductiva, planteamiento de hipótesis (predicción) con 18 ítems (evalúa si el examinado es capaz de extraer información de un texto y genera nueva información a partir de datos conocidos); 2. Clasificación, clasificación jerárquica, síntesis con 14 ítems (examina si el evaluado define palabras, identifica categorías, ordena proposiciones, etc.) y; 3. Razonamiento analógico, con 9 ítems, respectivamente (evalúa la capacidad de establecer relaciones de semejanza. entre términos de una proposición usando el argumento inductivo). 
Los factores antes mencionados fueron identificados pos sus autores, a partir de la aplicación del análisis factorial exploratorio y confirmatorio, en el marco del proceso de validación psicométrica del instrumento, que tuvo por finalidad conocer las variables latentes, no que subyacen al desempeño en la prueba; así como identificar si se obtenía la misma estructura factorial de los ítems de la primera fase de validación. Los resultados del análisis muestran ser favorables a la estructura de los factores mencionados, lo que indica que el modelo propuesto es válido, se concluye que la prueba presenta validez de constructo.

La confiabilidad de la prueba evidenció una consistencia interna elevada $(\mathrm{r}=$ .809) (coeficiente Alfa de Cronbach). Previo a los análisis de dificultad de los ítems así como de las correlaciones entre ítem y puntaje total, que llevó a un análisis de confiabilidad con los ítems seleccionados, arrojó finalmente un coeficiente de confiabilidad de $\mathrm{r}=.851$ verificando la alta consistencia interna de la prueba.

\section{Procedimiento}

La recogida de los datos se llevó a cabo en el mes de febrero de 2017, días antes del inicio del semestre 2017-I. Hubo un tiempo límite de 75 minutos en la administración del instrumento. Se informó a los participantes que su aceptación en la aplicación de los cuestionarios, indicaba su consentimiento oral para que los datos fueran utilizados para fines de su orientación en el aprendizaje, análisis estadísticos, siendo objeto posterior de presentación académica.

\section{RESULTADOS}

\section{Características descriptivas de la muestra}

La muestra de estudio estuvo constituida por 113 ingresantes universitarios de las carreras de ingeniería y arquitectura, pertenecientes a una universidad privada de la ciudad de Lima.Hay un $45.1 \%$ de evaluados del género femenino frente a un $54.9 \%$ del género masculino. Las edades son distribuidas en tres grupos de15 y 16 años (46\%), de 17 a 18 años (44.2\%) y mayores de 19 años (9.7\%). El análisis comparativo relacional entre habilidades del pensamiento y rendimiento académico fue realizado con 98 participantes que contaban con el promedio de nota final por haber cumplido con todas sus evaluaciones.

\section{Análisis de normalidad y estadísticos descriptivos de las variables de estudio}

Para establecer la distribución de las variables estudiadas se ha utilizado el test de normalidad de Kolmogorov-Smirnov Z, a fin de determinar el uso de los estadísticos paramétricos o no paramétricos. Los valores superiores a una significancia de 0.05 y 0.01 nos indican que hay una distribución normal.También son mostradas la Media (M), Desviación estándar (DS), puntajes mínimo y máximo, así como la confiabilidad Alpha de Cronbach total. 
En la Tabla 1 y Figura 1 podemos apreciar que la distribución de la muestra en Habilidades del Pensamiento y en sus factores es normal, con puntajes altos y bajos a los extremos de la curva.

\section{Tabla 1}

Análisis de normalidad de Kolmogorov-Smirnov Z y estadísticos descriptivos $(n=113)$.

\begin{tabular}{lc}
\hline & Habilidades del Pensamiento \\
\hline Kolmogorov-Smirnov Z & 1.20 \\
Significancia & 0.12 \\
Media & 22.61 \\
DS & 5.43 \\
Min & 9 \\
Max & 41 \\
Alpha de Cronbach & 0.71 \\
\hline p $>$.01: hay normalidad &
\end{tabular}

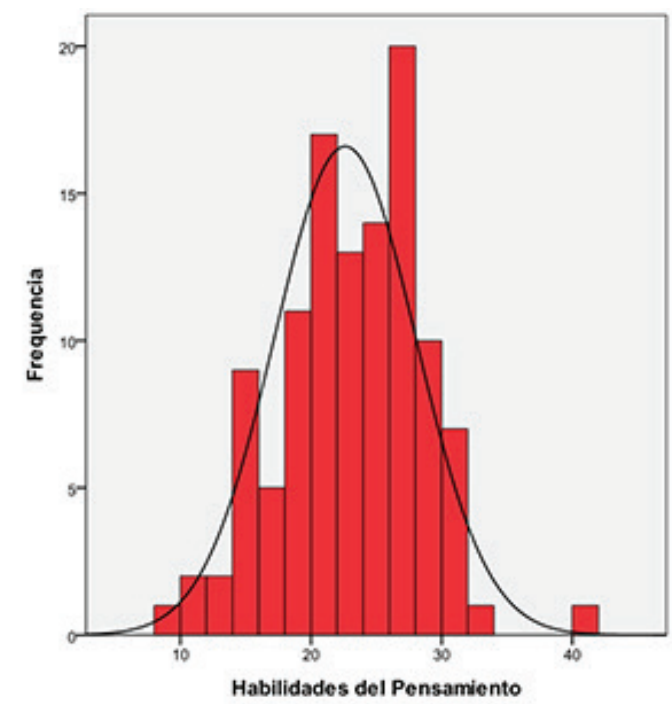

Figura 1. Puntajes de habilidades de Pensamiento.

Comparación de promedios por género, grupos de edad y especialidad en las variables de estudio.

Para establecer las diferencias en las Habilidades del pensamiento y sus factores se hizo uso de la t de Student, se observó que no existen diferencias estadísticamente significativas para ninguna de las tres variables $(\mathrm{p}>.05)$. 


\section{Distribución de Niveles de Habilidades del Pensamiento y sus factores de en la muestra de participantes total y por género.}

A continuación se distribuyen los niveles para el puntaje total de Habilidades del Pensamiento y para sus tres factores:

La distribución de los niveles se hace siguiendo a los autores de la Prueba (Tapia y Luna, 2010), considerando la muestra total $(\mathrm{n}=113)$ y por género ( $\mathrm{n}$ femenino $=51 ; \mathrm{n}$ masculino $=62$ ). También se ha incluido el estadístico Chi cuadrado para establecer la existencia de asociación significativa entre niveles y género.

\section{Distribución de Niveles de Habilidades del Pensamiento.}

En la Tabla 2 se presenta la distribución de los niveles de Habilidades del Pensamiento. No se han encontrado asociaciones significativas. Sin embargo, podemos apreciar que la muestra se distribuye mayoritariamente (53\%) en el nivel Normal Inferior y Normal, mientras que un $20.4 \%$ se ubica en el Promedio y un $26.65 \%$ se distribuye en los niveles Normal superior y Superior.

Tabla 2

Distribución de los niveles de Habilidades del Pensamiento en la muestra total y por género $(n=113)$.

\begin{tabular}{ccccccccc}
\hline Género & & Inferior & $\begin{array}{c}\text { Normal } \\
\text { Inferior }\end{array}$ & Normal & $\begin{array}{c}\text { Normal } \\
\text { Superior }\end{array}$ & Superior & $\begin{array}{c}\text { Chi } \\
\text { cuadrado }\end{array}$ & Sig \\
\hline Muestra total & $\mathrm{n}$ & 30 & 30 & 23 & 21 & 9 & .532 & .970 \\
& $\%$ & 26.5 & 26.5 & 20.4 & 18.6 & 8.0 & & \\
Femenino & $\mathrm{n}$ & 14 & 13 & 10 & 9 & 5 & & \\
& $\%$ & 12.4 & 11.5 & 8.8 & 8.0 & 4.4 & & \\
Masculino & $\mathrm{n}$ & 16 & 17 & 13 & 12 & 4 & & \\
& $\%$ & 14.2 & 15.0 & 11.5 & 10.6 & 3.5 & & \\
\hline
\end{tabular}

$\mathrm{p}<.05^{*}:$ diferencia significativa $\mathrm{p}<.01^{* *}:$ diferencia muy significativa

\section{Distribución de Niveles de los Factores de Habilidades del Pensamiento}

En la Tabla 3 se aprecia la distribución de los niveles del Factor 1: Inferencia lógicadeductiva e inductiva, del Factor 2: Clasificación, clasificación jerárquica, síntesis y del Factor 3:Razonamiento analógico.

En el Factor 1, no se han encontrado asociaciones significativas. Sin embargo, podemos apreciar que la muestra se distribuye mayoritariamente en el nivel Normal (31\%), seguido de un $15.9 \%$ en el nivel Normal inferior y un $19.6 \%$ en el Normal superior. En los extremos de la distribución vemos que un $19.5 \%$ se ubica en el nivel Inferior y un $14.2 \%$ en el nivel Superior. 
En el Factor 2, tampoco se han encontrado asociaciones significativas. Sin embargo, podemos apreciar que la muestra total se distribuye mayoritariamente en el nivel Inferior (42.5\%) y Normal inferior (24.8\%). A nivel Promedio un 15.9\% logra ubicarse, mientras que solo un $16.8 \%$ logra ubicarse entre los niveles Normal Superior y Superior.

En el Factor 3, no se han encontrado asociaciones significativas,aunque se aprecia que la muestra se distribuye mayoritariamente en el nivel Normal Superior y Normal (55.7\%), mientras que un 38\% lo hace entre los niveles Normal inferior e Inferior, respectivamente. El 6.2\% logra ubicase en el nivel Superior.

Tabla 3

Distribución de los niveles de los factores de habilidad del pensamiento en la muestra total y por género $(n=113)$.

\begin{tabular}{lcccccccc}
\hline Género & & Inferior & $\begin{array}{c}\text { Normal } \\
\text { Inferior }\end{array}$ & Normal & $\begin{array}{c}\text { Normal } \\
\text { Superior }\end{array}$ & Superior & $\begin{array}{c}\text { Chi } \\
\text { cuadrado }\end{array}$ & Sig \\
\hline Muestra total & $\mathrm{n}$ & 22 & 18 & 35 & 22 & 16 & 2.72 & .61 \\
& $\%$ & 19.5 & 15.9 & 31 & 19.5 & 14.2 & & \\
Femenino & $\mathrm{n}$ & 8 & 11 & 16 & 9 & 7 & & \\
& $\%$ & 7.1 & 9.7 & 14.2 & 8 & 6.2 & \\
Masculino & $\mathrm{n}$ & 14 & 7 & 19 & 13 & 9 & \\
& $\%$ & 12.4 & 6.2 & 16.8 & 11.5 & 8 & \\
\hline \multirow{2}{*}{ Muestra total } & $\mathrm{n}$ & 48 & 28 & 18 & 11 & 8 & 8.05 & \\
& $\%$ & 42.5 & 24.8 & 15.9 & 9.7 & 7.1 & \\
Femenino & $\mathrm{n}$ & 17 & 13 & 8 & 6 & 7 & \\
& $\%$ & 15 & 11.5 & 7.1 & 5.3 & 6.2 & \\
Masculino & $\mathrm{n}$ & 31 & 15 & 10 & 5 & 1 & \\
& $\%$ & 27.4 & 13.3 & 8.8 & 4.4 & 0.9 & \\
\hline
\end{tabular}

Factor 3: Razonamiento analógico en la muestra total y por género

\begin{tabular}{lcccccccc}
\hline Muestra total & $\mathrm{N}$ & 26 & 17 & 31 & 32 & 7 & 7.56 & .11 \\
& $\%$ & 23 & 15 & 27.4 & 28.3 & 6.2 & & \\
Femenino & $\mathrm{N}$ & 13 & 3 & 15 & 15 & 5 & & \\
& $\%$ & 11.5 & 2.7 & 13.3 & 13.3 & 4.4 & \\
Masculino & $\mathrm{N}$ & 13 & 14 & 16 & 17 & 2 & & \\
& $\%$ & 11.5 & 12.4 & 14.2 & 15 & 1.8 & & \\
\hline
\end{tabular}

$\mathrm{p}<.05 *$ : diferencia significativa $\mathrm{p}<.01 * *$ : diferencia muy significativa 


\section{Análisis comparativo de la relación entre Habilidades del Pensamiento (puntaje global) y sus factores con la nota final $(n=98)$.}

En la Tabla 4 presentamos la relación entre el rendimiento académico, dado por la nota final obtenida por los participantes en una escala de 0 a 20. Cabe mencionar nuevamente que la muestra total fue de 113 participantes; sin embargo, este análisis se realiza con los participantes que contaban con promedio o nota final al término del semestre académico.

Hemos hecho uso de la correlación de Pearson para establecer esta relación y podemos apreciar que todas las correlaciones son positivas y significativas al .05 de probabilidad, encontrando un $\mathrm{r}$ : 0.53 de los puntajes de la prueba total con el promedio final. Es decir se verificó la existencia de una correlación positiva entre el rendimiento académico y las Habilidades del Pensamiento (puntaje total) entre los participantes del estudio.

Cuando observamos esta relación por factores de Habilidad del Pensamiento, podemos observar lo siguiente: en relación al Factor 1 (Inferencia lógica deductiva e inductiva, planteamiento de hipótesis), se observa un r: 0.51 , seguida de un r: 0.36 en el Factor 2 (Clasificación, clasificación jerárquica, síntesis) y de un r: 0.29 en el factor 3: Razonamiento analógico.En estos casos también se aprecia correlación significativa entre los factores y el rendimiento académico.

\section{Tabla 4}

Relación entre los puntajes de Habilidades del pensamiento y las notas finales en los participantes con promedio final $(n=98)$.

\begin{tabular}{lcc}
\hline & & Promedio final \\
\hline Inferencia lógica deductiva e inductiva, planteamiento & $\mathrm{r}$ & 0.51 \\
de hipótesis & $\mathrm{sig}$ & $0.00^{* *}$ \\
Clasificación, clasificación jerárquica, síntesis & $\mathrm{r}$ & 0.36 \\
& $\mathrm{sig}$ & $0.00^{* *}$ \\
Razonamiento analógico & $\mathrm{r}$ & 0.29 \\
& $\mathrm{sig}$ & $0.00^{* *}$ \\
Habilidades del Pensamiento & $\mathrm{r}$ & 0.53 \\
& $\mathrm{sig}$ & $0.00^{* *}$ \\
\hline
\end{tabular}

$* \mathrm{p}<.05$ : correlación significativa

\section{DISCUSIÓN}

Los estudiantes que inician un primer ciclo universitario, egresados recientes del nivel secundario, no están al cien por ciento definidos en sus habilidades del pensamiento, de ahí que resulta importante que se propenda a identificar en ellos sus niveles de desarrollo en dichas habilidades, para tomar decisiones respecto de su optimización a través del proceso de enseñanza-aprendizaje. 
En el proceso de formación profesional, resulta importante prestar atención a la potenciación de las habilidades del pensamiento para que se produzca la adquisición y apropiación de habilidades de pensamiento de orden superior, de pensamiento reflexivo, de combinación de ideas y de asunción de riesgos mentales, para establecer conexiones, evaluar los pasos seguidos y llegar a realizar conclusiones razonadas, a partir de experiencias en las especialidades concretas en las que se están formando profesionalmente.

Los resultados hallados en el presente estudio, nos muestra que a nivel de las habilidades del pensamiento (puntajes globales), un 53\% de los participantes se distribuye mayoritariamente en el nivel Normal Inferior y Normal, solo un $26.65 \%$ se distribuye en los niveles Normal superior y Superior, y un $20.4 \%$ se ubica en el Promedio. Dichos resultados, con los hallazgos respecto a la existencia de una asociación positiva moderada con el rendimiento académico, nos sugiere la necesidad de potenciar las habilidades del pensamiento evaluadas en cada una de las asignaturas de carrera y de humanidades que cursan los estudiantes en el primer ciclo de estudios. Ello podría ser punto de inicio para incentivar el rendimiento estimulando las habilidades de pensamiento.

Muelas y Navarro (2014), han mostrado correlaciones significativas entre estrategias de aprendizaje y el rendimiento académico en estudiantes de $1^{\circ}$ de bachillerato; particularmente en la asignatura de lengua, en la cual el rendimiento se relaciona significativamente con las estrategias de codificación (con una correlación del 0.45 y una significatividad del 0.00 ) y de recuperación (con una correlación del 0.56 y una significatividad del 0.00 ), en tanto que con matemáticas, la correlación significativa se da en la estrategia de codificación (con una correlación del 0.49 y una significatividad del 0.00). Observa que el desarrollo de las habilidades de pensamiento en los estudiantes, mediante el empleo de estrategias de aprendizaje, influye significativamente en su rendimiento académico.

Sánchez (2002), en el marco de la presentación de un modelo integrado de investigación y desarrollo aplicable a la construcción, implantación y evaluación de proyectos para la enseñanza y transferencia de habilidades del pensamiento, comunicó que en su revisión de instituciones universitarias, se implantaron de tres a cinco niveles del curso "Desarrollo de habilidades del pensamiento" dirigido a la enseñanza directa de procesos de pensamiento como materia curricular, y se entrenaba a los profesores para que ofrecieran clases de pensamiento e instigaran la transferencia de los procesos a la enseñanza y el aprendizaje de otras disciplinas del plan de estudios y situaciones de la vida diaria.

Entre los factores que constituyen las habilidades del pensamiento, tal cual se han medido, muestran a los participantes menos hábiles en la organización del conocimiento, que comprende utilizar las operaciones de clasificación, clasificación jerárquica y síntesis (Factor 2). Sabemos que mediante la clasificación se realizan 
dos tipos de operaciones mentales: agrupar conjuntos de personas elementos de diversa índole en clases; y, establecer categorías conceptuales (Sánchez,1995).

El acto de clasificar es la base de la definición de conceptos; mediante este proceso, al identificar las características del mismo y señalar ejemplos, la definición de conceptos a través de la clasificación, amplía la posibilidad de autoaprendizaje y eleva el nivel de abstracción de la persona. Por otro lado, el acto de realizar síntesis que es un proceso complementario al análisis, consiste en integrar todos los aspectos analizados y realizar una conclusión, resumen u opinión personal a partir de los elementos que se examinaron anteriormente.

En las habilidades deInferencia lógica deductiva e inductiva, planteamiento de hipótesis (Factor 1) y razonamiento analógico (Factor 3), por presentar los participantes una mayor distribución de su desempeño entrelos niveles Normal superior yNormal, se sugiere reforzar dichas habilidades en las asignaturas que más las requieran.

Con relación a la no existencia de diferencias entre las habilidades de pensamiento y sus factores con el género, edad, los resultados concuerdan con las de otros investigadores. No hemos encontrado referencias con relación a las diferencias por especialidades en las ingenierías, en el proceso de formación profesional.

Finalmente, esperamos que nuestra investigación contribuya al estudio de las habilidades del pensamiento en grupos de estudiantes universitarios, ya que basándonos en los diferentes estudios que hemos citado creemos en la utilidad de realizar este tipo de estudios, sobre todo en universitarios que están iniciando una carrera universitaria, esto ayudará tanto a los docentes como a los alumnos a mejorar la metodología en el aprendizaje y se pueda diseñar proyectos de cambio e intervención de las habilidades en los alumnos con miras a mejorar tanto el rendimiento académico como las formas del aprendizaje de las asignaturas impartidas en la universidad.

También consideramosimportante potenciar gradualmente los procesos que subyacen a las habilidades del pensamiento que han mostrado menor desarrollo, para que en ciclos académicos superiores el estudiante universitario adquiera con mayor facilidad conocimientos y habilidades más complejas, que loconduzca hacia la metacognición, para lograr tener mayor conciencia de cómo aprende. En tal sentido, los docentes universitarios podrían proporcionar experiencias de aprendizaje y aplicar estrategias didácticas para potenciar los procesos pertinentes, por ello surge como necesidad enfoques innovadores y modelos de estructuras en la educación universitaria, para afrontar retos asociados al desarrollo de las habilidades esenciales del pensamiento. 


\section{REFERENCIAS BIBLIOGRÁFICAS}

Feuerstein, R. (1990). The theory of structural modifiability. In B. Presseisen (Ed.), Learning and thinking styles: Classroom interaction. Washington: National Education Associations.

Feuerstein, R. (1997). Teoría de la modificabilidad cognitiva estructural. En: Martínez, J. M.; Tebar, L.; Rivera, J. A (Dirección) ¿Es modificable la inteligencia? Edit. Bruño.

Gardner, H. (1983). Frames of mind: The theory of multiple intelligences. New York: Basic Books. Gardner, H. (1985). The mind's new science: A history of the cognitive revolution. New York: Basic Books

Goleman, D. (1986). Inteligencia emocional. Barcelona: Kairós.

Muelas y Navarro (2014). Learning Strategies and Academic Achievement. Procedia Social and Behavioral Sciences 165 ( 2015 ) 217 - 221.

Sánchez, M. (1985). Teaching thinking processes. En D. N. Perkins, J. Lockhead y J.C. Bishop (Eds.), Thinking: The Second International Conference (pp. 413-430). Hillsdale, NJ: Lawrence Erlbaum Associates.

Sánchez, M. (1985). Using critical thinking principles as a guide to college-level instruction. Teaching of Psychology 22, (1), 72-74.

Sánchez, M. (1992). Programa Desarrollo de Habilidades de Pensamiento. Revista Intercontinental de Psicología y Educación 5 (2), 207-236.

Sánchez, M. (2002). La investigación sobre el desarrollo y la enseñanza de las habilidades de pensamiento, Revista electrónica de investigación educativa 4(1), 128-159.

Sternberg, R. (1985). Beyond I. Q. A triarchic theory of human intelligence.Cambridge: Cambridge University Press.

Sternberg, R. (1987). Intelligence applied. Understanding and increasing your intellectual skills. Nueva York: W. H. Freeman and Co.

Tapia, V. y Luna, J. (2010). Validación de una prueba de habilidades de pensamiento para alumnos de cuarto y quinto de secundaria y primer año de universidad. Revista de Investigación en psicología, 13 (2). 17-59.

Velásquez, B; Remolina, N; Calle, A. (2013).Habilidades de pensamiento como estrategia de aprendizaje para los estudiantes universitarios. Revista de Investigaciones UNAD. $12(2), 23-41$. 